\title{
Optimized combinatorial pMHC class II multimer labeling for precision immune monitoring of tumor-specific CD4 T cells in patients
}

\author{
Georg Alexander Rockinger (D , ,,2 Philippe Guillaume, ${ }^{1,2}$ Amélie Cachot, ${ }^{1,2}$ \\ Margaux Saillard, ${ }^{1,2}$ Daniel E Speiser, ${ }^{1}$ Georges Coukos, ${ }^{1,2}$ Alexandre Harari, ${ }^{1,2}$ \\ Pedro J Romero, ${ }^{1}$ Julien Schmidt, ${ }^{1,2}$ Camilla Jandus (i) ${ }^{1,2}$
}

To cite: Rockinger GA, Guillaume P, Cachot A, et al. Optimized combinatorial pMHC class II multimer labeling for precision immune monitoring of tumor-specific CD4 T cells in patients. Journal for ImmunoTherapy of Cancer 2020;8:e000435. doi:10.1136/ jitc-2019-000435

Accepted 12 February 2020

Check for updates

(C) Author(s) (or their employer(s)) 2020. Re-use permitted under CC BY-NC. No commercial re-use. See rights and permissions. Published by BMJ.

${ }^{1}$ Department of Oncology UNIL CHUV, University of Lausanne, Epalinges, Switzerland

${ }^{2}$ Ludwig Institute for Cancer Research, Lausanne Branch of Immunology, Epalinges, Switzerland

\section{Correspondence to} Dr Camilla Jandus, Department of Pathology and Immunology, Faculty of Medicine, University of Geneva, Geneva, Switzerland; camilla.jandus@unige.ch

\begin{abstract}
Background With immunotherapy gaining increasing approval for treatment of different tumor types, scientists rely on cutting edge methods for the monitoring of immune responses and biomarker development in patients. Due to the lack of tools to efficiently detect rare circulating human tumor-specific CD4 T cells, their characterization in patients still remains very limited.

Methods We have used combinatorial staining strategies with peptide major histocompatibility complex class II (pMHCII) multimer constructs of different alleles to establish an optimized staining procedure for in vitro and direct ex-vivo visualization of tumor-specific CD4 T cells, in patient samples. Furthermore, we have generated reversible multimers to achieve optimal cell staining and yet disassemble prior to in vitro cell expansion, thus preventing activation induced cell death. Results We observed a vastly improved detection of tumor-specific, viral-specific and bacterial-specific cells with our optimization methods compared with the nonoptimized staining procedure. By increasing the variety of fluorochromes used to label the pMHCII multimers, we were also able to increase the parallel detection of different specificities within one sample, including antigen-specific CD8 T cells. A decrease in cell viability was observed when using the full optimization method, but this was mitigated by the removal of neuraminidase and the use of reversible multimers.

Conclusion This new optimized staining procedure represents an advance toward better detection and analysis of antigen-specific CD4 T cells. It should facilitate state-of-the art precision monitoring of tumor-specific CD4 T cells and contribute to accelerate the use and the targeting of these cells in cancer immunotherapy.
\end{abstract}

\section{INTRODUCTION}

The identification of numerous tumor associated antigens and neo-antigens recognized by tumor reactive $T$ cells in recent years has fueled the rapid progress in the field of cancer immunotherapy. Their exquisite specificity has been exploited in the exploration of several immunotherapeutic strategies including cancer vaccines, adoptive $\mathrm{T}$ cell transfer (ACT) and the use of immunomodulatory agents. To date, cytotoxic CD8 T cells have been the major focus of cancer immunotherapy due to their capacity to directly recognize and eliminate cancer cells. ${ }^{1}$ However, CD4 $\mathrm{T}$ cells are becoming more appreciated due to new evidence emerging on their crucial role in cell-based therapies, peptide-based vaccination and in the recognition of neo-antigens. ${ }^{2-4}$ Nonetheless, the low frequencies of circulating tumor-specific CD4 $\mathrm{T}$ cells, their low affinity $\mathrm{T}$ cell receptors (TCRs) and the lack of tools to adequately detect and isolate them have all hindered their characterization at the antigen specific level and their clinical exploitation remains limited.

The direct measurement of antigenspecific $\mathrm{T}$ cell responses is done through the use of recombinant major histocompatibility complex (MHC) multimers assembled with antigenic peptides major histocompatibility complex (pMHC) used in multiparameter flow cytometry or mass cytometry. Major advances have been made in quantitative specific $\mathrm{T}$ cell frequency assays with MHC class I peptide multimers (pMHCI), including high multiplex panels of more than 1000 pMHCI DNA-barcoded multimers. ${ }^{5}$ However, little progress has been made with pMHC class II (pMHCII) molecules. Several hurdles might explain this gap, such as low affinity TCR specific for MHC class II complexes, ${ }^{6}$ modest coreceptor contribution to pMHCII binding avidity, ${ }^{7-9}$ the conformational diversity of pMHCII complexes ${ }^{10}$ the high polymorphism of HLA-DP, HLA-DQ, HLA-DR molecules and the poor quality of pMHC class II multimers related to technical difficulties in their generation. ${ }^{6}$ In regard to 
the latter, recent advances have improved the yields and stability of pMHCII multimers. These include the addition of a leucine zipper instead of the transmembrane and cytoplasmic domains preventing dissociation of the alpha and beta chains, ${ }^{11}$ the addition of a C-terminal His-tag, allowing gentle affinity purification of the complexes, ${ }^{10}$ the use of conditional peptide tags improving the degree of peptide loading ${ }^{12}$ and the linking of the peptide to the $\beta$ chain via a long and flexible linker. ${ }^{13}$ In turn, these advances have led to improved identification of antigen-specific CD4 T cells, mainly in the context of viral infections or autoimmunity, ${ }^{14}$ whereas very little data are available to date on the detection of human tumorspecific CD4 T cells in patients.

Here, we generated pMHCII multimers and reversible pMHCII NTAmers ${ }^{15}$ and used them in combination with several molecules known to impact on TCR-pMHC interactions to optimize antigen-specific CD4 $\mathrm{T}$ cell detection and enumeration. These included galectin-3, a sugarbinding lectin that is capable of forming a lattice on the cell surface and binding to various glycosylated cell surface receptors such as TCRs ${ }^{16}$ and impairing TCRpMHC interaction. This effect is reversed by the addition of N-acetyl-D-lactosamine (LacNAc), a competitive galectin-binder. ${ }^{17}{ }^{18}$ Furthermore, the removal of sialic acid may increase ${ }^{171920}$ multimer staining with pMHC multimers.

We show that the combined use of neuraminidase, dasatinib, LacNAc as well as an anti-fluorochrome antibodies $(\mathrm{Ab})^{13}{ }^{21}$ improves the specific labeling of rare human tumor-specific CD4 T cells. This optimized multimer labeling assay enables the concomitant immunomonitoring of tumor-antigen specific CD4 and CD8 T cells from a single tube, thereby reducing the sample size needed. Furthermore, we report on the successful direct ex-vivo detection of tumor-specific CD4 T cells as well as the isolation and clonal expansion of these cells, for TCRengineering and adoptive cell therapy approaches.

\section{METHODS}

\section{Patients, blood samples and ethical approval statement}

Peripheral blood from patients with stage III/IV melanoma was obtained from the Department of Oncology, University Hospital (CHUV), Lausanne, Switzerland, on written informed consent based on the study protocol (NCT00112242) approved by the IRB and Swissmedic. ${ }^{3}$ The patients had been subjected to a vaccination protocol with the 30 amino acid peptide LSP (long synthetic peptide) NY-ESO- ${ }_{79-108}$, administered subcutaneously in combination with CpG-B (CpG-7909/PF-3512676) and Montanide ISA-51, with or without low dose interleukin-2. Blood was diluted with phosphate-buffered saline (PBS) or RPMI 1640 (Invitrogen, Paisley, UK), mononuclear cells were purified by centrifugation over Ficoll-Plaque TM Plus (Amersham Bioscience, Uppsala, Sweden) and washed three times with RPMI 1640. Lymphocytes were directly cryopreserved in 50\% RPMI (Gibco/Thermo Fisher Scientific, Massachusetts, USA), 40\% FCS (PAA Laboratories/GE Healthcare, Illinois, USA), and $10 \%$ dimethyl sulfoxide (DMSO) freezing medium (SigmaAldrich, Missouri, USA). Vials of $10 \times 10^{6}$ peripheral blood mononuclear cells (PBMC) from patients with melanoma were stored in liquid nitrogen.

\section{Peptide epitopes}

Peptides were synthesized by the Peptide and Tetramer Core Facility, UNIL-CHUV, Epalinges, Switzerland, by standard solid phase peptide synthesis on a multiple peptide synthesizer (Intavis, Germany). All peptides were $>90 \%$ pure as indicated by ultra performance liquid chromatography/mass spectrometry (UPLCMS) analysis. Lyophilized peptides were diluted in pure DMSO at $10 \mathrm{mg} / \mathrm{mL}$ or aliquots of $1 \mathrm{mg} / \mathrm{mL}$ in $10 \%$ DMSO were prepared and stored at $-80^{\circ} \mathrm{C}$. The peptide epitopes and their restricting HLA elements are listed in table 1.

\begin{tabular}{|c|c|c|c|c|}
\hline Peptide name & Peptide sequence & Peptide origin & HLA restriction & HLA \% \\
\hline $\mathrm{HA}_{307-319}$ & PKYVKQNTLKLAT & Hemagglutinin & HLA-DRB1*07:01 & 25 \\
\hline $\mathrm{HA}_{307-319}$ & PKYVKQNTLKLAT & Hemagglutinin & HLA-DRB1*04:01 & 24 \\
\hline MAGE-A3 ${ }_{243-258}$ & KKLLTQHFVQENYLEY & Melanoma-associated antigen 3 & HLA-DPB1*04:01 & 75 \\
\hline $\mathrm{TT}_{947-960}$ & FNNFTVSFWLRVPK & Tetanus toxoid & HLA-DPB1*04:01 & 75 \\
\hline Melan- $A_{26-35(A 27 L)}$ & ELALIGILTV & MART-1 & HLA-A*02:01 & 44 \\
\hline $\mathrm{EBV}_{280-288}$ & GLCTLVAML & BMLF-1 & HLA-A*02:01 & 44 \\
\hline $\mathrm{CMV}_{495-503}$ & NLVPMVATV & pp65 & HLA-A*02:01 & 44 \\
\hline
\end{tabular}




\section{Generation of pMHC II multimers}

Peptide-MHC II multimers were produced by the Peptide and Tetramer Core Facility, UNIL-CHUV, Epalinges, Switzerland. The extracellular region of the HLA-DR/DP $\alpha$ and HLA-DR/DP $\beta$ chains were separately cloned into pMT BiP\V5-His A (ThermoFisher scientific). The alpha chain construct harbors the acidic leucine zipper and terminates by a tandem His-tag (HHHHHHGGGSGG GSGSHHHHHH). The beta chain construct contains the basic leucine zipper and terminates with AviTag sequence. To generate cell lines expressing HLA-DR or HLA-DP, the two plasmids with a third plasmid conferring puromycin resistance, were cotransfected into Drosophila S2 cells using Cellfectin (ThermoFisher Scientific) according to the manufacturer protocol. Protein expression was induced by addition of $1 \mathrm{mM}$ CuSO4. MHC class II molecules were purified from supernatants with Chelating Sepharose FF (Merck). Peptide loading was performed in $100 \mathrm{mM}$ sodium citrate, $\mathrm{pH} 6.0$ with $100 \mu \mathrm{M}$ peptide at $37^{\circ} \mathrm{C}$ for 24 hours, buffer-exchanged on a HiPrep 26/10 desalting column (Merck) into AviTag buffer and subsequently biotinylated with the BirA enzyme according to the manufacturer instructions (Avidity, Denver, Colorado, USA). Biotinylated MHC class II-peptide complexes were purified on a HisTrap HP column (Merck).

Peptide-MHC II monomers were multimerized using streptavidin-conjugated dyes and then aliquoted and kept at $-80^{\circ} \mathrm{C}$. Reversible pMHC II multimers (NTAmers) were prepared similarly other than the use of a proprietary streptavidin-NTA conjugate.

\section{Generation of $\mathrm{T}$ cell clones}

PBMC from patients with HLAs of interest were thawed and CD4 $\mathrm{T}$ cells enriched using anti-CD4 microbeads and MiniMACS magnetic separation columns (Miltenyi Biotec, Bergisch Gladbach, Germany). These were then stimulated with autologous irradiated $\mathrm{CD} 4^{-} \mathrm{T}$ cells in the presence of a pool of peptides ( $5 \mu \mathrm{M}$ each), as indicated. After 2 days of culture, $100 \mu \mathrm{L}$ of RPMI 1640 containing $8 \%$ of human serum, $2 \mathrm{mM}$ glutamine, $1 \%$ ( $\mathrm{vol} / \mathrm{vol}$ ) nonessential amino acids, $50 \mu \mathrm{M}$ 23-mercaptoethanol, penicillin $(50 \mathrm{U} / \mathrm{mL})$ and streptomycin $(50 \mu \mathrm{g} / \mathrm{mL})(\mathrm{R} 8$ media) was replaced with fresh media containing a final concentration of $100 \mathrm{IU} / \mathrm{mL}$ of human recombinant $(\mathrm{hr})$ IL-2. After 10 days of in vitro expansion, cultures were tested for the presence of antigen-specific CD4 T cells with pMHCII multimers loaded with the same peptide used in the stimulation process. The detection of these cells was performed with the standard multimer staining process: the phycoerythrin (PE) or APC-conjugated multimers were added to the cells at a final concentration of $10 \mu \mathrm{g}$ / $\mathrm{mL}$ in a volume of $25 \mu \mathrm{L}$ of $\mathrm{R} 8$ media and incubated for $45 \mathrm{~min}$ at room temperature (RT). Twenty minutes before the end of the multimer incubation period, $25 \mu \mathrm{L}$ of the following antibody cocktail was added directly to each sample without washing: anti-CD3 AF700 (clone HIT3a, Biolegend, London, UK), anti-CD4 FITC (clone RPA-T4, Biolegend) and LIVE/DEAD fixable dead cell stain (Vivid, Invitrogen, California, USA) diluted 1:800 in R8 media. Where indicated, anti-CD45RA ECD (clone 2H4LDH11LDB9, Beckman Coulter, California, USA) and anti-CCR7 BV421 (clone G043H7, Biolegend) were used. Samples were analyzed with the BD LSR II Flow Cytometry Analyzer (Becton Dickinson, New Jersey, USA). The data were analyzed using FlowJo software (FlowJo). The positive cultures were then sorted using FACS cytometers either Aria IIU or the Aria III (Becton Dickinson) using the same staining protocol.

Individual, sorted, epitope-specific $\mathrm{T}$ cells were stimulated with $1 \mu \mathrm{g} / \mathrm{mL}$ phytohemagglutinin (PHA) (Thermo Fisher Scientific) in the presence of irradiated (30 Gy) allogeneic PBMC feeder cells $\left(10 \times 10^{4}\right.$ per well $)$ and hrIL-2 $(100 \mathrm{IU} / \mathrm{mL})$ and plated in Terasaki plates for clone generation. The remaining sorted cells were stimulated and cultured in a 96-well plate as a bulk of epitope specific cells. $\mathrm{T}$ cells were cultured for a minimum of 2 weeks postrestimulation before being used for experiments or frozen.

\section{Optimized and reversible multimer staining}

For optimized CD4 T cell multimer staining, the cells were plated in 96-well $\mathrm{V}$ bottom plates and centrifuged at $1500 \mathrm{rpm}$ (453g on Eppendorf 5810R). After removing the supernatant, the cells were resuspended in $100 \mu \mathrm{L}$ of R8 media containing LacNAc (Sigma-Aldrich) at a final concentration of $5 \mathrm{mM}$ and incubated at $37^{\circ} \mathrm{C}$ for a period of 2 hours. Cells were washed with PBS, centrifuged and resuspended in $100 \mu \mathrm{L}$ of PBS containing dasatinib (Axon Medchem, Virginia, USA) and neuraminidase from Clostridium perfringens (C. welchii) (SigmaAldrich) at respective final concentrations of $50 \mathrm{nM}$ and $0.7 \mathrm{U} / \mathrm{mL}$ and then incubated for $30 \mathrm{~min}$ at $37^{\circ} \mathrm{C}$. After a further PBS cell wash, standard multimer staining was performed (see above) after which $0.5 \mu \mathrm{g}(10 \mu \mathrm{g} /$ $\mathrm{mL}$ ) of either mouse anti-PE unconjugated $\mathrm{Ab}$ (clone PE001, Biolegend) or mouse anti-APC unconjugated Ab (clone APC003, Biolegend) was added depending on the labeling fluorochrome bound to the multimer and incubated for $20 \mathrm{~min}$ on ice in the dark. Cells were washed and resuspended in PBS and analyzed with either the BD LSR II Flow Cytometry Analyzer (Becton Dickinson) or with the CytoFLEX S Flow Cytometry Analyzer (Becton Dickinson). When reversible multimers were used in this protocol, cells were treated with Imidazole at $100 \mathrm{mM}$ in a volume of $100 \mu \mathrm{L}$ of PBS for $2 \mathrm{~min}$ at $4^{\circ} \mathrm{C}$, either at the end of the staining procedure or immediately after single cell sorting.

\section{Imaging flow cytometry}

Following sample thawing, clonal cells were split into two wells of a 96-well $\mathrm{V}$ bottom plate. Half of the wells were subjected to the standard multimer staining procedure whereas the other half were treated with the optimized staining procedure (OSP). The cells were stained with multimers and cell surface markers. To avoid spillover between fluorochromes, the panel was modified to 
contain: the PE labeled specific multimer, anti-CD3 APC (clone UCHT1, Beckman Coulter, California, USA), anti-CD4 BV605 (clone OXT4, Biolegend) and LIVE/ DEAD fixable dead cell stain (Vivid, Invitrogen). The remaining two wells were stained with the Mouse AntiHuman TCR $\alpha \beta$ PE (clone T10B9.1A-31, Becton Dickinson) for $45 \mathrm{~min}$ in $25 \mu \mathrm{L}$ of R8 media at RT. Twenty minutes before the end of the incubation period $25 \mu \mathrm{L}$ of R8 media containing the previously mentioned CD3 APC, CD4 BV605 and LIVE/DEAD fixable dead cell stain was added and incubated at RT. All samples were analysed with the Amnis Imaging flow cytometry (Luminex, TX, USA) and data processed with the IDEAS Software (Luminex, TX, USA).

\section{Evaluation of cell viability}

CD4 $\mathrm{T}$ cell clones were seeded in a 96-well $\mathrm{V}$ bottom plate and subjected to standard or optimized multimer staining procedures and put in culture in a round bottom 96-well plate in R8 media containing $100 \mathrm{IU} / \mathrm{mL}$ of hrIL-2. At time points 6 hour, 24 hours and 48 hours, the cells were collected and stained for viability markers. Cells were washed with PBS and resuspended in $25 \mu \mathrm{L}$ of PBS containing anti-CD3 AF700 (clone HIT3a, Biolegend), anti-CD4 FITC (clone RPA-T4, Biolegend) and LIVE/ DEAD fixable dead cell stain (Vivid, Invitrogen) diluted 1:800 in PBS. The cells were incubated at RT for $30 \mathrm{~min}$ and washed with PBS. They were resuspended in $25 \mu \mathrm{L}$ of AnnexinV buffer 1X with AnnexinV-PE (Becton Dickinson) and incubated for $20 \mathrm{~min}$ at $4^{\circ} \mathrm{C}$. Cells were then washed and resuspended in AnnexinV buffer 1X and analyzed with the CytoFLEX S Flow Cytometry Analyzer (Becton Dickinson).

\section{Functional evaluation}

For the evaluation of the secreted cytokines on specific stimulation, CD4 T cell clones were plated in a 96-well U-shaped plate, left unstimulated, stimulated with $5 \mu \mathrm{M}$ of specific $\mathrm{HA}_{307-319}$ or NY-ESO-1 $1_{87-99}$ peptides or with $5 \mu \mathrm{M}$ of an irrelevant peptide. The cells were incubated $\mathrm{O} / \mathrm{N}$ with the addition of $2.5 \mu \mathrm{g} / \mathrm{mL}$ of Brefeldin A. The cells were then collected and transferred to a 96-well V-shaped plate for flow cytometry staining. The cells were first incubated for $30 \mathrm{~min}$ at RT with anti-CD3 BV421 (clone UCHT1, Biolegend), anti-CD4 FITC (clone RPA-T4, Biolegend) and LIVE/DEAD fixable dead cell stain (Vivid, Invitrogen) diluted 1:800 in PBS. Cells were then fixed and permeabilized following the recommendations provided with the eBioscience Foxp3/Transcription Factor Staining Buffer Set. Cells were washed twice with the permeabilization buffer $1 \mathrm{X}$ provided with the kit and then incubated for a period of $30 \mathrm{~min}$ at RT with the anti-IFN $\gamma$ PE (clone B27, Biolegend) and the anti-TNF $\alpha$ AF700 (clone Mab11, Becton Dickinson) diluted in permeabilization buffer 1X. The cells were washed and analyzed with the CytoFLEX S Flow Cytometry Analyzer (Becton Dickinson).

\section{Statistical analysis}

Statistical tests used were the Wilcoxon matched-pairs signed-ranks test and two-way analysis of variance followed by Tukey's multiple comparisons.

\section{RESULTS \\ Optimal staining of antigen-specific CD4 T cells by pMHC class II multimers is achieved at room temperature}

To systematically optimize pMHCII multimer labeling of specific CD4 T cells, we first evaluated the effect of temperature on the labeling of a collection of human $\mathrm{CD} 4 \mathrm{~T}$ cell clones of tumor-antigen and viral specificities by design multimers, as illustrated in figure $1 \mathrm{~A}$. We compared the staining efficiency at $4^{\circ} \mathrm{C}, 15^{\circ} \mathrm{C}$, RT and $37^{\circ} \mathrm{C}$. Although distinct labeling and of HLA-DR7/ NY-ESO-1 $1_{87-99}$ specific CD4 T cell clones with the HLA-DR7/ NY-ESO-1 ${ }_{87-99}$ multimer was obtained at all temperatures, maximal mean fluorescence intensity (MFI) was apparent at RT compared with other temperatures (figure 1B-D). The increase in MFI was not accompanied by an increase in background staining, as assessed in criss-cross experiments with irrelevant clones (figure 1E). Similar results were obtained using viral specific clones $\left(\mathrm{HA}_{307-319}\right.$ specific clones, restricted by HLA-DR7) (figure 1F). These results suggest that optimal pMHCII multimer staining requires exposure of CD4 T cells to multimers at RT.

\section{The use of optimizing molecules improves pMHCII multimer staining of human antigen-specific CD4 T cells}

To determine whether the multimer staining quality could be further improved, a set of candidate molecules individually and in combination was included in the staining procedure. CD4 T cell clones specific for NY-ESO- ${ }_{87-99}$ or $\mathrm{HA}_{307-319}$ were pretreated with neuraminidase, dasatinib or both, before multimer staining. In addition to the various cell treatments, a secondary antibody recognizing the phycoerythrin fluorochrome incorporated into the multimers was added prior to analysis. Addition of either neuraminidase or dasatinib significantly increased the fluorescent signal. Moreover, the addition of both had an additive effect (figure 2A,B). The addition of the secondary antibody further increased the detected MFI, with no impact on background staining of an irrelevant clone (figure 2C,D).

Next, knowing that LacNAc, a competitive binder of galectin, affects TCR mobility on the cell membrane, we tested its effect on multimer staining. Clones were incubated with multimers after treatment with LacNAc alone, with the combination of optimizing molecules (dasatinib, neuraminidase and secondary $\mathrm{Ab}$ ) or with the optimizing molecules plus LacNAc. The use of LacNAc alone did not improve the multimer staining. However, when used in combination with the other optimizing molecules, it resulted in the highest specific MFI signals (figure 2E). We used the Amnis Imaging flow cytometry technology to directly visualize the multimer distribution on the cell surface of clones labeled with either the standard 
A

B
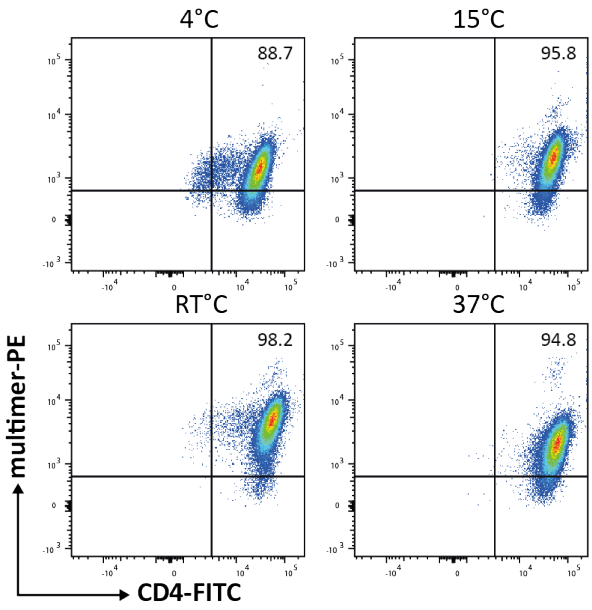

E

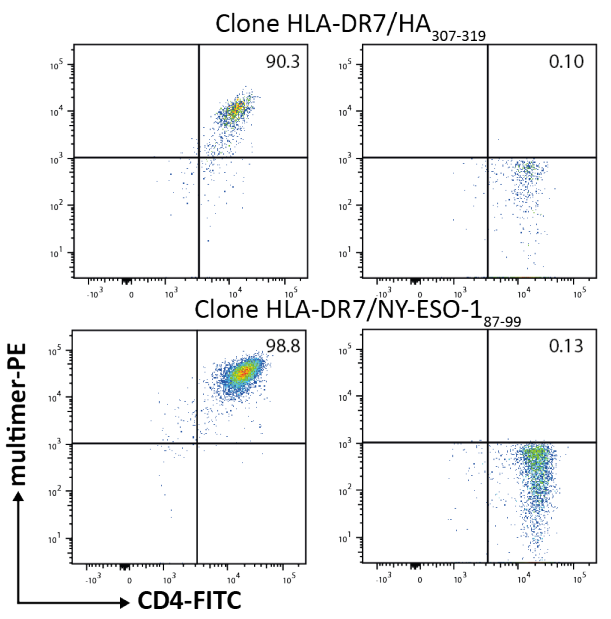

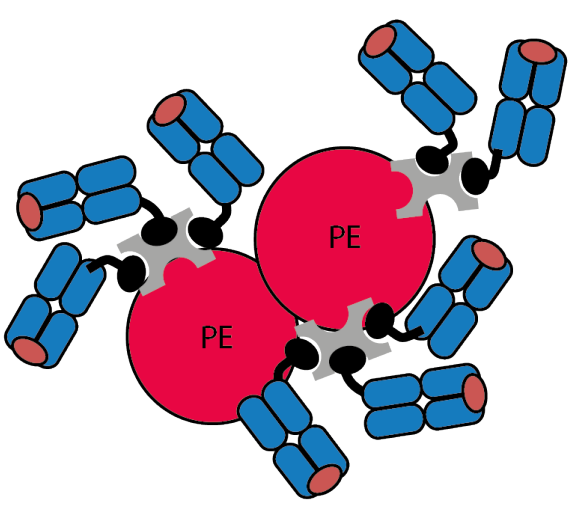

C

D
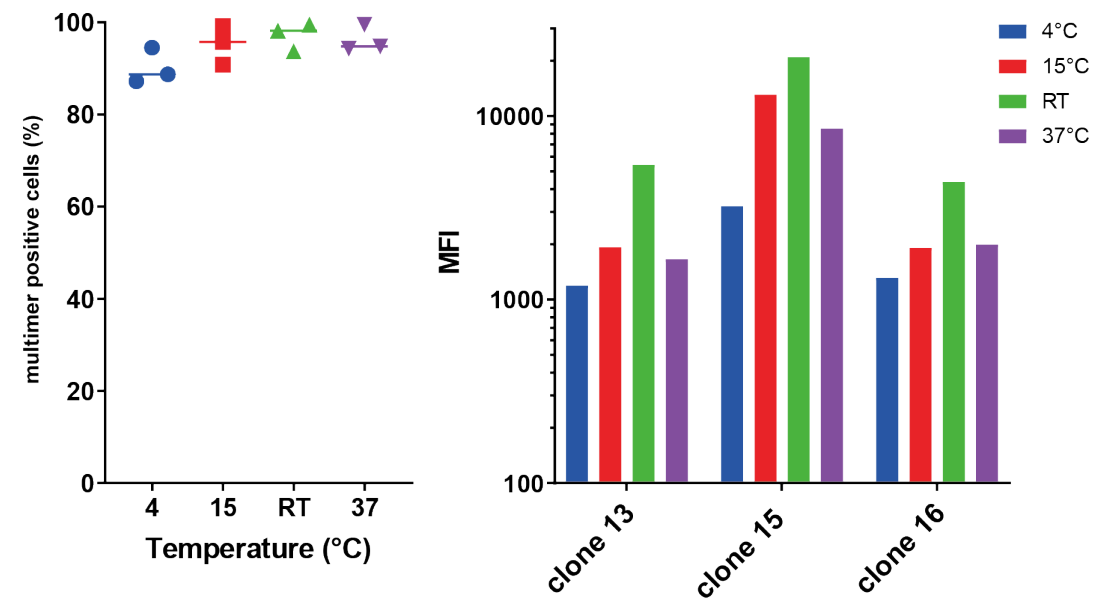

$\mathbf{F}$

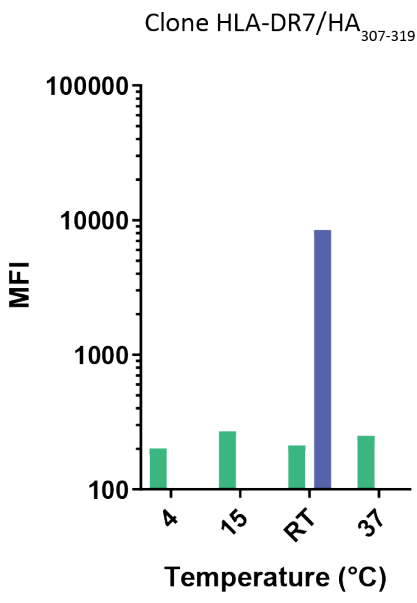

Figure 1 Temperature optimization for pMHCll multimer staining. (A) Basic illustration of pMHCll multimers. Multimers were composed of a fluorochrome backbone (PE in the example, red), an average of 2-3 streptavidin complexes (grey) bound to biotin (black)-labeled pMHC complexes (blue), loaded with the peptide of interest (brown). (B) Representative dot plots illustrating the impact of the temperature on multimer staining. Staining was performed on specific CD4 T cell clones using an HLA-DR7 multimer loaded with the NY-ESO-1 ${ }_{87-99}$ peptide. Dot plots are gated on live lymphocytes, CD3 ${ }^{+}$. (C,D) Summary of the results obtained on three different clones for the frequency of multimer positive cells (C) and the MFI (D). (E) Representative

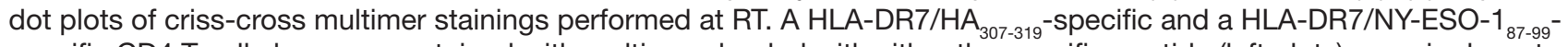
specific CD4 T cell clones were stained with multimers loaded with either the specific peptide (left plots) or an irrelevant peptide (right plots). (F) Histogram representation of the MFIs obtained using an irrelevant (staining at all temperatures) or the specific multimer (only used at RT). The clone $\mathrm{HA}_{307-319}$ was stained with the specific multimer (in blue) or with the nonspecific NYESO-1 ${ }_{87-99}$ /DR7 multimer (in green). Vice versa, the NY-ESO-1 ${ }_{87-99}$ clone was stained with the specific multimer (in green) and the nonspecific $\mathrm{HA}_{307-319}$ /DR7 multimer (in blue). MFI, mean fluorescence intensity; pMHCII, peptide MHC class II; RT, room temperature. 
A

B
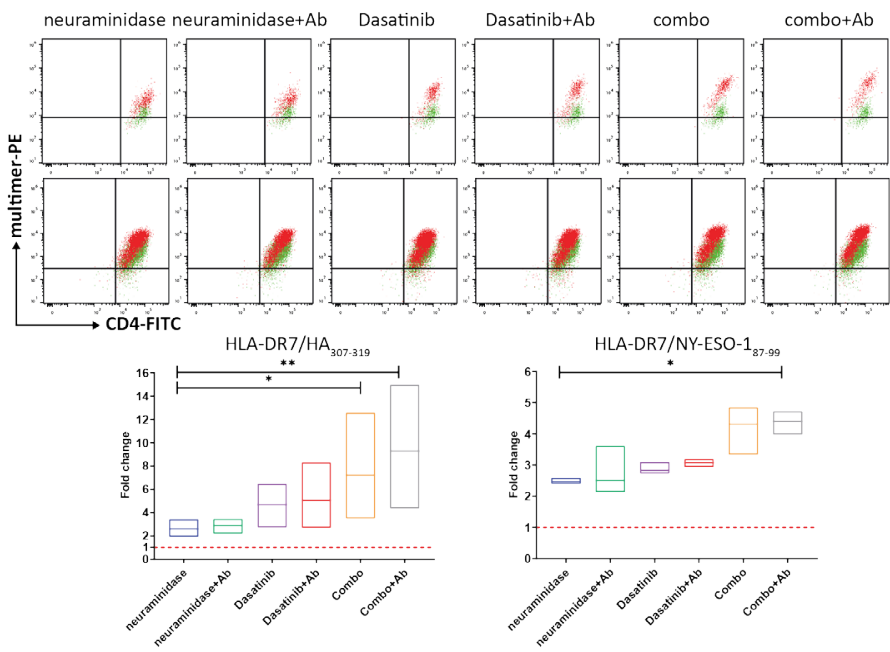

C
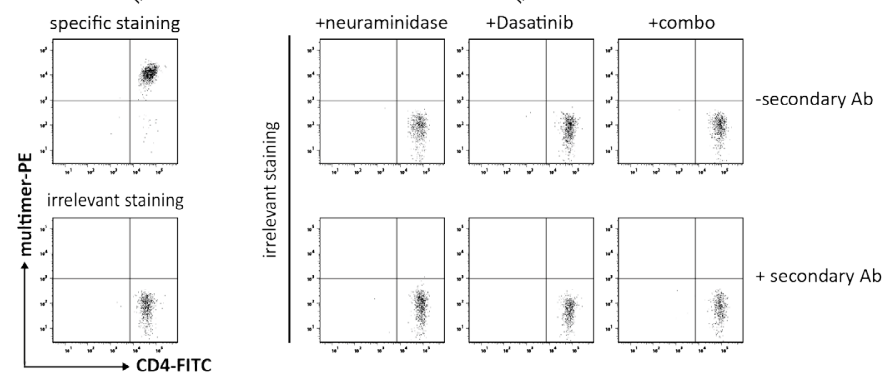

D
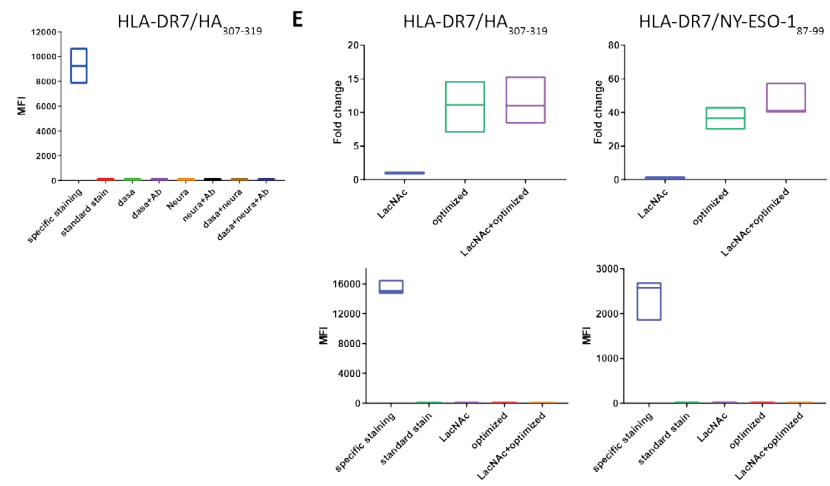

F
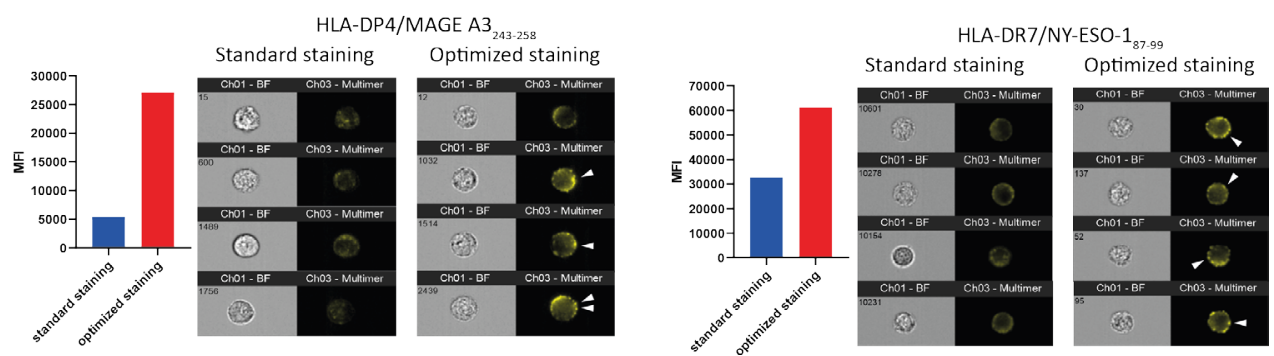

Figure 2 Improvement of the standard pMHCII multimer staining procedure. (A) Representative dot plots showing the impact of the indicated molecules tested either individually or in combinations, with or without secondary cross-linking Ab (red) overlaid on the standard staining procedure (green). Optimization was tested on a HLA-DR7/HA ${ }_{307-319}$ clone (top) and on a HLA-DR7/ NY-ESO-1 $1_{87-99}$ clone (bottom). Dot plots are gated on live lymphocytes, CD3 ${ }^{+}$. (B) Fold change representation of the impact on MFI increase by each optimizing condition for both antigen-specificities $(n=4)$. (C) Representative dot plots of nonspecific multimer binding by a HLA-DR7/NY-ESO-1 ${ }_{87-99}$ multimer on a $\mathrm{HA}_{307-319}$-specific clone subjected to the optimization treatments. Dot plots are gated on live lymphocytes, $\mathrm{CD}^{+}$. (D) MFI representation of nonspecific multimer staining in all optimization conditions for all clones $\mathrm{HA}_{307-319}$ specific tested $(n=3)$. (E) Contribution of the LacNAc molecule to the optimization of multimer staining, tested on a HLA-DR7/HA ${ }_{307-319}$-specific clone (left) and on HLA-DR7/NY-ESO-1 ${ }_{87-99}$ clone (right). MFI representation of nonspecific multimer staining in all optimization conditions is shown on the same clones on the bottom panels. (F) Imagestream visualization of the increased staining intensity of CD4 T cell clones by multimers under optimization conditions. Clone HLADP4/MAGE-A3 ${ }_{243-258}$ (left) and HLA-DR7/NY-ESO-1 ${ }_{87-99}$ (right) were stained with either standard or with optimized staining procedures. Both MFI and cell images are represented. White arrows illustrate clusters of multimers. MFI, mean fluorescence intensity; pMHCII, peptide MHC class II. 
or OSP. This confirmed the increased MFI with the OSP compared with the standard procedure and revealed clustering of fluorescently labeled multimers only with the OSP protocol (figure 2F). Together, these results indicate that the pretreatment of $\mathrm{T}$ cells with the optimizing molecules LacNAc, neuraminidase and dasatinib followed by incubation with a secondary cross-linking $\mathrm{Ab}$, leads to optimal multimer labeling as assessed by flow cytometry.

\section{Optimizing molecules allow improved staining across multiple antigen specificities and HLA restrictions}

In order to validate the OSP across multiple antigenspecificities, clones specific for HLA-DR7/HA $307-319$, HLA-DR7/NY-ESO-1 ${ }_{87-99}$, HLA-DR4/HA ${ }_{307-319}$, HLA-DP4/ MAGE-A3 ${ }_{111-125}$, HLA-DP4/MAGE-A3 ${ }_{243-258}$ or HLA-DP4/ tetanus toxoid (TT) ${ }_{947-960}$ were labeled with either the standard procedure or the OSP (figure 3A). An increase in MFI signals using the OSP was consistently observed compared with the standard procedure. No specific signal was observed when an irrelevant multimer was used (figure 3A).

For immune monitoring purposes, improved identification of tumor-specific CD4 T cells is necessary directly ex vivo, in patient-derived PBMC samples. Ex-vivo samples from HLA-DR7+ and HLA-DP4+ patients with stage III/ IV melanoma were labeled with either the standard procedure or the OSP. Increased frequencies of tumor antigen-specific $\mathrm{CD} 4 \mathrm{~T}$ cells could be measured when using the OSP compared with the standard procedure (figure 3B,C) with no background staining difference between the two (figure 3B-D).

Samples from stage III-IV melanoma patients prevaccination and postvaccination with the LSP NY-ESO- $1_{79-108}$ were stained with the HLA-DR7/NY-ESO- $1_{87-99}$ or HLADR4/HA H07-319 multimers. $\mathrm{HA}_{307-319}$ specific cells were detected at a higher frequency when using the OSP compared with the standard staining procedure, but their frequency did not vary during vaccination (figure $3 \mathrm{E}$ ). An increase in NY-ESO- $1_{87-99}$ specific CD4 T cell frequencies was observed following vaccination and accentuated when using the OSP (figure 3E). Furthermore, with the OSP, a small population of NY-ESO- $1_{87-99}$-specific CD4 T cells was readily detectable in samples prevaccination. The detection of larger proportions of viral-specific and tumorspecific cells using the OSP was further demonstrated after in-vitro expansion of CD4 T cells from patients' postvaccination (figure $3 \mathrm{G}$ ).

The use of the OSP in healthy donor (HD) samples resulted in the detection of higher frequencies of NY-ESO$1_{87-99}$ specific CD4 T cells compared with standard staining (figure $3 \mathrm{H}$ ). The NY-ESO-1 $1_{87-99}$ specific cells were predominantly of a naïve (N) phenotype (figure 3I). Further, the HDs showed low frequencies of $\mathrm{HA}_{307-319}$ specific cells with a mixed differentiation phenotype. Overall, mostly effector memory (EM) and central memory (CM) cells were stained with the standard NY-ESO- $1_{87}$. ${ }_{99}$ or $\mathrm{HA}_{307-319}$ specific multimer staining (figure 3F-I). A larger proportion of $\mathrm{N}$ cells as well as $\mathrm{CM}$ and $\mathrm{EM}$ and terminally differentiated effector memory cells (EMRA) were detected with the OSP. NY-ESO- $1_{87-99}$-specific cells displayed an evolution from mostly $\mathrm{N}$ cells prevaccination in patients' samples toward more CM and EM differentiated cells postvaccination (figure $3 \mathrm{~F}$ ).

Overall, these results confirm the efficacy of OSP monitoring of antigen-specific CD4 $\mathrm{T}$ cells, also directly ex vivo in HD and patient peripheral blood samples.

\section{Combinatorial labeling of antigen-specific CD4 and CD8 T cells using OSP in patient samples}

Due to the limited amount of biological material available from patients, it would be advantageous if multiple specificities could be investigated within the same individual sample. For this purpose, a cocktail of pMHCI and pMHCII multimers labeled with either one or a combination of two fluorochromes as described in figure $4 \mathrm{~A}$ was used. First, the feasibility of combinatorial staining for CD4 and CD8 T cells was verified by spiking CD4 and CD8 $\mathrm{T}$ cell clones of the specificities indicated in figure $4 \mathrm{~A}$, into PBMCs. All the specific clones could be detected with the combination of multimers (figure 4B). The OSP allowed for a much sharper discrimination between specific and negative cells in the CD4 $\mathrm{T}$ cell population than the standard labeling procedure (figure 4B,C). For CD8 $\mathrm{T}$ cells, the optimization was effective in the detection of $\mathrm{EBV}_{280-288}, \mathrm{Flu}_{58-66}$ and Melan- $\mathrm{A}_{26-35}$ specific cells but not in the case of $\mathrm{CMV}_{495-503}$ and NY-ESO-1 $1_{157-165}$ specific cells (figure 4B,C).

Peripheral blood lymphocyte samples from HLA-DR $7^{+}$, HLA-DP4 $^{+}$and HLA-A2 ${ }^{+}$patients with melanoma were used to evaluate the efficiency of combinatorial multimer labeling with and without the optimization procedure directly on ex-vivo cells. Multiple antigen specificities were detected within the samples which benefited from the optimization, with a higher frequency of specific cells detected and a higher staining intensity achieved when using the OSP (figure 4D). These results demonstrate the feasibility of integrating the OSP with combinatorial multimer labeling for sensitive detection of both class I and class II-restricted $\mathrm{T}$ cells of multiple specificities within a single patient sample.

\section{The usage of reversible multimers improves cell viability of tumor-specific CD4 T cells on subsequent expansion}

Besides its applicability for direct ex-vivo immune monitoring, the OSP might be advantageous in sorting specific cells for in vitro expansion for adoptive cell transfer therapy. In this regard, the impact of the OSP on CD4 $T$ cell viability was evaluated using HLA-DP4/MAGE$\mathrm{A} 3_{243-258}$ restricted clones with either an intermediate or high MFI (figure 5A). Once the labeling procedure was complete, the cells from each condition were seeded and cultured for periods of 6 hours, 24 hours and 48 hours before viability assessment. With the intermediate MFI clones, substantial cell death was apparent in cloned $\mathrm{T}$ cells after OSP treatment, irrespective of the presence of the multimer (figure 5B). In contrast, the cloned T cells 
A

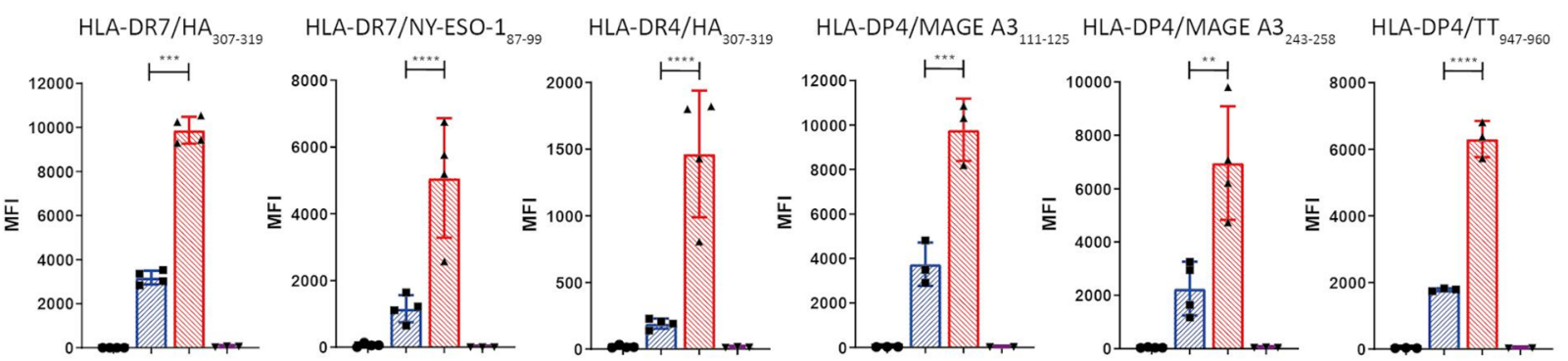

Key: No multimer, Standard staining, Optimized staining, Non-specific staining

B

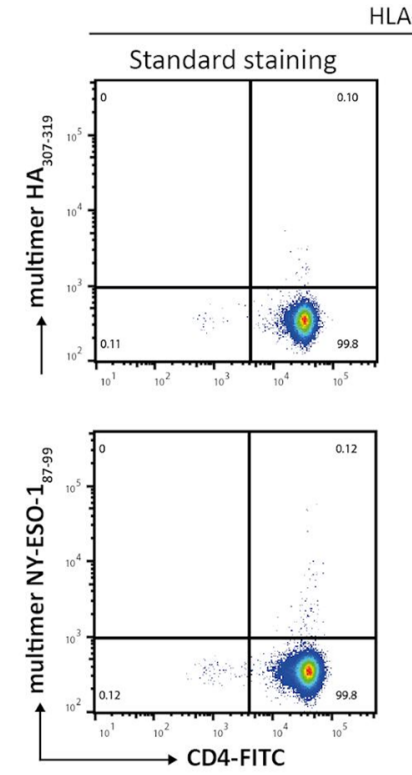

Optimized staining
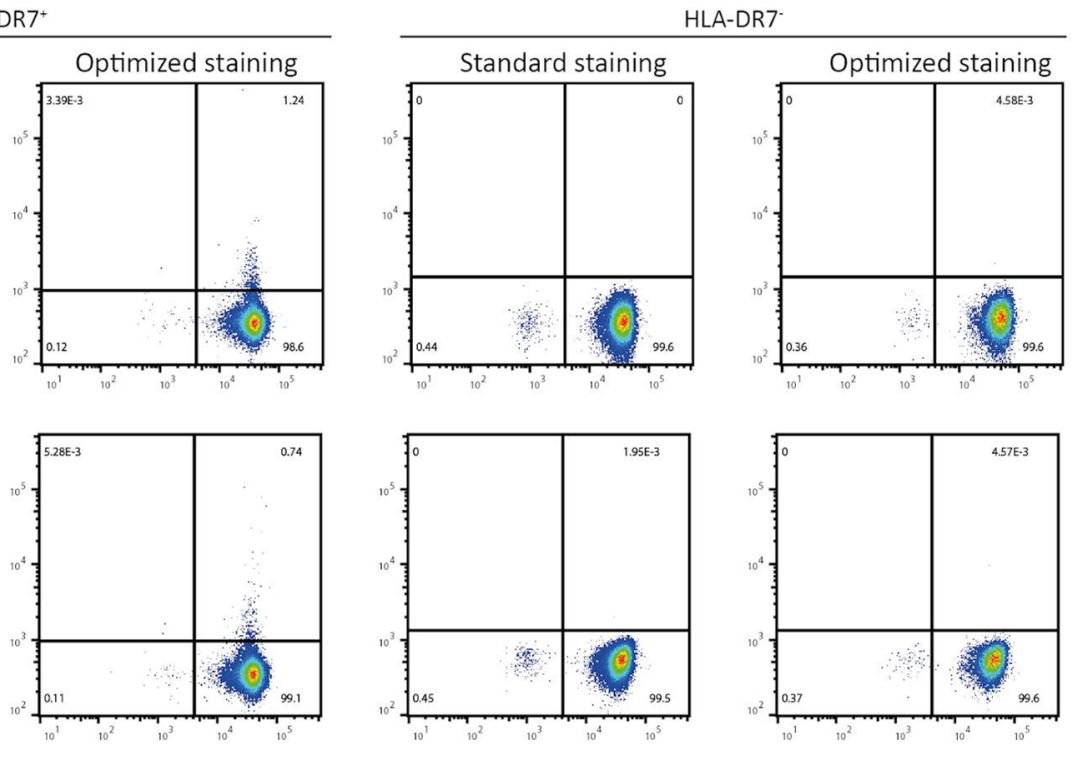

C

$\mathrm{HLA}-\mathrm{DR} 7 / \mathrm{HA}_{307-319}$
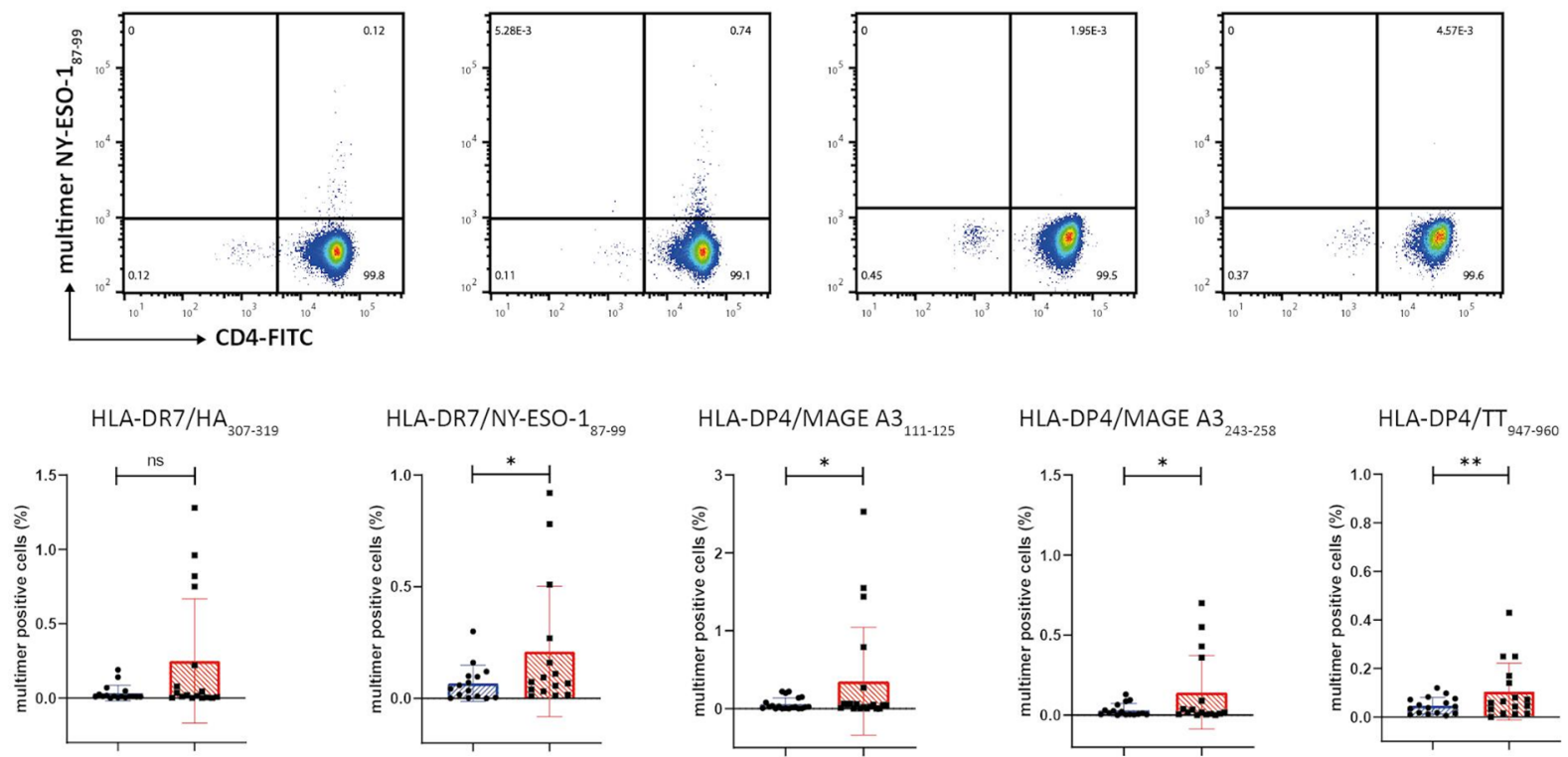

D

HLA-DR7/HA $307-319$

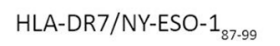

HLA-DP4/MAGE A3 ${ }_{111-125}$

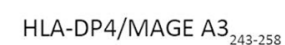

HLA-DP4/TT $947-960$
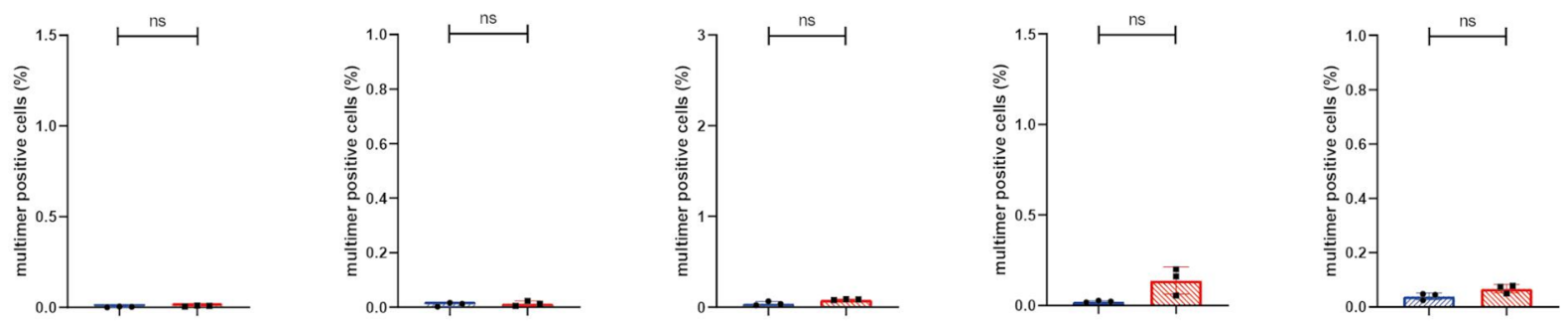

Key: Standard staining, Optimized staining

Figure 3 (Continued) 
$\mathbf{E}$
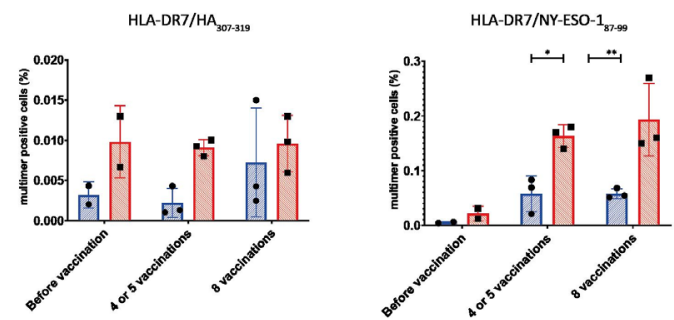

$\mathbf{F}$
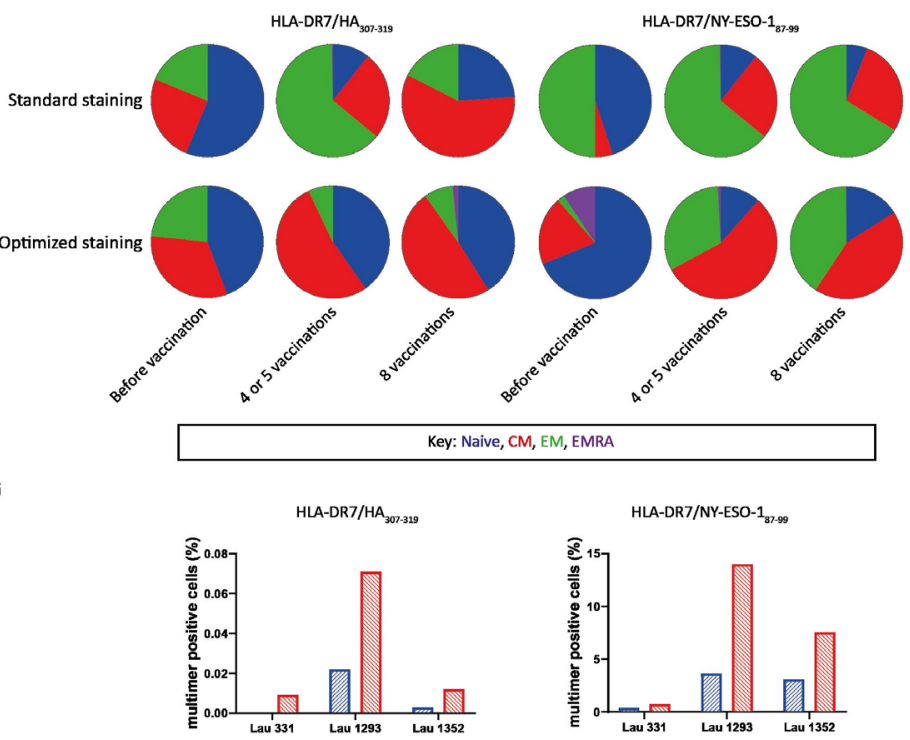

H
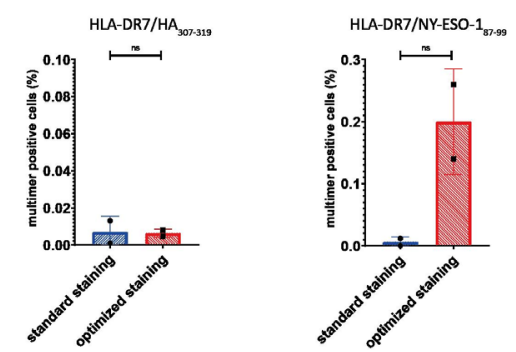

I

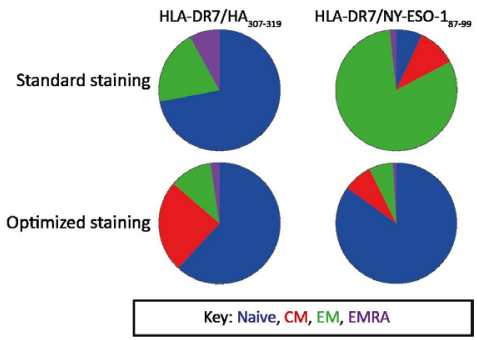

Figure 3 In-vitro and ex-vivo improvement of multimer staining across multiple specificities. (A) Clones specific for HLADR7/HA ${ }_{307-319}(n=4)$, HLA-DR7/NY-ESO-1 $1_{87-99}(n=4)$, HLA-DR4/HA ${ }_{307-319}(n=4)$, HLA-DP4/MAGE-A3 ${ }_{111-125}(n=3)$, HLA-DP4/ MAGE-A3 $_{243-258}(\mathrm{n}=4)$ and HLA-DP4/TT $947-960(\mathrm{n}=3)$ were stained using standard (blue) and optimized staining procedures (red). For negative controls, the staining was performed in the absence of the multimer (black) or with a nonspecific multimer (purple). (B) Representative dot plots of ex-vivo staining to detect HLA-DR7/HA ${ }_{307-319}$ (top) and HLA-DR7/NY-ESO-1 ${ }_{87-99}$ (bottom) specific CD4 T cells in DR7 ${ }^{+}$patient samples (left) or in mismatched DR7 ${ }^{-}$patient samples (right) using standard or the OSP. Dot plots are gated on live lymphocytes, $\mathrm{CD}^{+}$. (C) Detection of epitope-specific CD4 T cells in ex-vivo samples by standard (blue) and optimized multimer staining (red). Specificities investigated include HLA-DR7/HA ${ }_{307-319}(n=17)$, HLA-DR7/NY-ESO-1 $1_{87-99}(n=15)$, HLA-DP4/MAGE-A3 ${ }_{111-125}(n=20)$, HLA-DP4/MAGE-A3 ${ }_{243-258}(n=16)$, HLA-DP4/ $\Pi_{947-960}^{307-319}(n=16)$. Frequencies of multimer positive cells among total $\mathrm{CD} 4^{+} \mathrm{CD}^{+}$cells. (D) HLA-mismatched ex-vivo samples were stained using multimers for each one of the previously mentioned specificities, comparing the standard (blue) to the optimized staining procedure (red). Frequencies of multimer positive cells among total $\mathrm{CD}^{+} \mathrm{CD}^{+}$cells $(n=3)$. (E) Samples from patients with melanoma, either prevaccination $(n=2)$ or post-4 to 5 vaccinations $(n=3)$ or post- 8 vaccinations $(n=3)$ were stained with HLA-DR7/HA $A_{307-319}$ or HLA-DR7/NYESO-1 $1_{87-99}$ multimers comparing the standard (blue) to the optimized staining procedure (red). (F) The phenotype of multimer positive cells in standard stained or optimized multimer stained populations was evaluated with the addition of the CCR7 and CD45RA markers. Naïve (blue), CM (red), EM (green) and EMRAs (purple) frequencies were analyzed at the different timepoints during vaccination ( $n=2, n=3, n=3$, respectively, for each time point). (G) Standard multimer staining (blue) or optimized multimer procedure (red) post-in vitro stimulation with $\mathrm{HA}_{307-319}$ or NY-ESO-1 ${ }_{87-99}$ peptides of samples from patients wiht melanoma postvaccination. (H) HLA-DR7/HA ${ }_{307-319}$ or HLA-DR7/NY-ESO-1 $1_{87-99}$ multimer staining for the detection of specific cells in two HLA-DR7 healthy donors $(n=2)$. Standard protocol (blue) is compared with the optimized protocol (blue). (I) Representation of the CD4 T cell phenotype among the multimer positive cells of healthy donors stained with the HLA-DR7/HA ${ }_{307-319}$ or HLA-DR7/ NY-ESO- $1_{87-99}$ multimers $(n=2)$. Represented in the pie-chart are the naïve population (blue), CM (red), EM (green) and EMRA (purple). CM, central memory; EM, effector memory; EMRA, terminally differentiated effector memory cells; OSP, optimized staining procedure. 
A

B
NY-ESO- 87-99 $_{\text {DR7-PE/BV421 }}$

$\mathrm{HA}_{307-319}$ /DR7-PE/APC

MAGE A3 ${ }_{111-125} / D P 4-A P C$

MAGE A3 ${ }_{243-25} /$ DP4-PE

$\Pi_{947-960} / \mathrm{DP} 4-\mathrm{APC} / \mathrm{BV} 605$

EBV ${ }_{280-288} / A 2-P E-T e x a s$ Red

$\mathrm{CMV}_{\text {495-503 }} / \mathrm{A2}-\mathrm{BV} 421$

$\mathrm{Flu}_{58-66} / \mathrm{A2}-\mathrm{BV} 711$

NY-ESO-1 $1_{157-165} / \mathrm{A} 2-\mathrm{PE}$

Melan- ${ }_{26-35} / A 2-A P C$

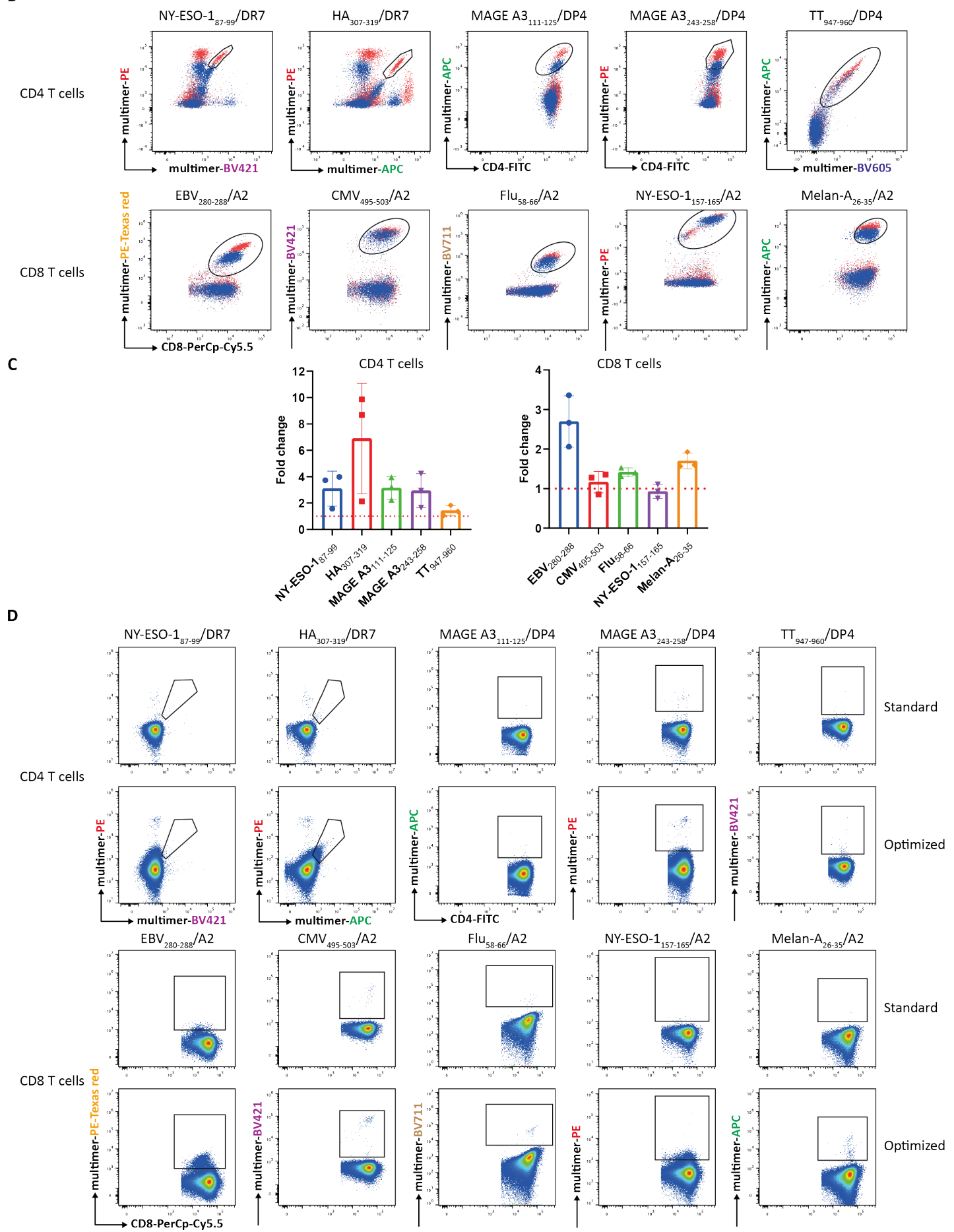

Figure 4 Combinatorial optimized staining for improved in vitro and ex-vivo detection of multiple antigen-specificities. (A) Panel of fluorescent multimers used in the experimental mix. On the left list of $\mathrm{pMHCll}$ multimers and on the right of pMHCl multimers. (B) Representative dot plots of the detection of multiple antigen-specific T cells spiked in PBMC stained either with the standard (blue) or optimized multimer procedure (red). Gated on live CD3 ${ }^{+} \mathrm{CD} 4^{+}$or $\mathrm{CD} 8^{+}$cells. (C) Fold change representation of the increased MFI comparing standard and optimized staining procedures for the different antigen specificities. CD4 T cells are represented on the left and CD8 T cells on the right $(n=3)$. (D) Representative dot plots of a combinatorial ex-vivo multimer staining on $\mathrm{HLA}-\mathrm{DR} 7^{+} / \mathrm{HLA}-\mathrm{DP} 4^{+} / \mathrm{HLA}-\mathrm{A} 2^{+}$patients with melanoma. Both standard and optimized staining are represented. Gated on live, $\mathrm{CD}^{+} \mathrm{CD}^{+}$or $\mathrm{CD} 8^{+}$cells. pMHCll, peptide MHC class II. 

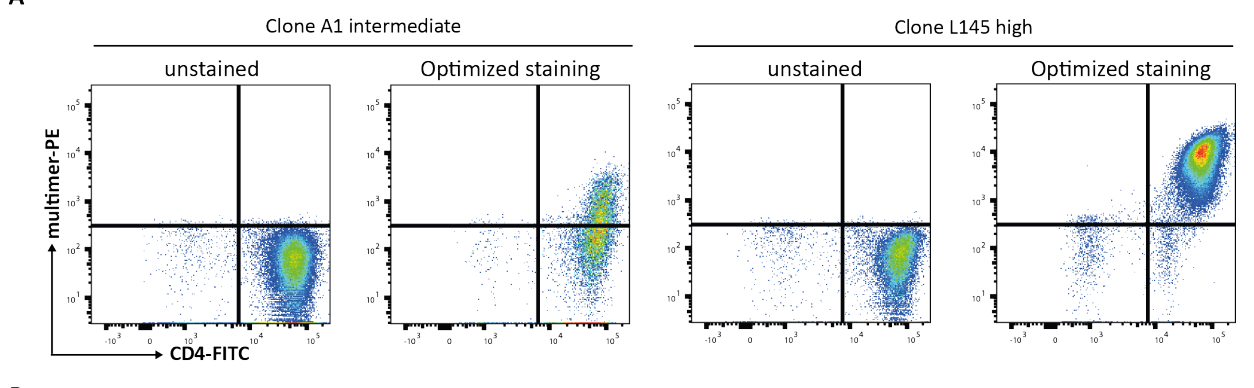

B
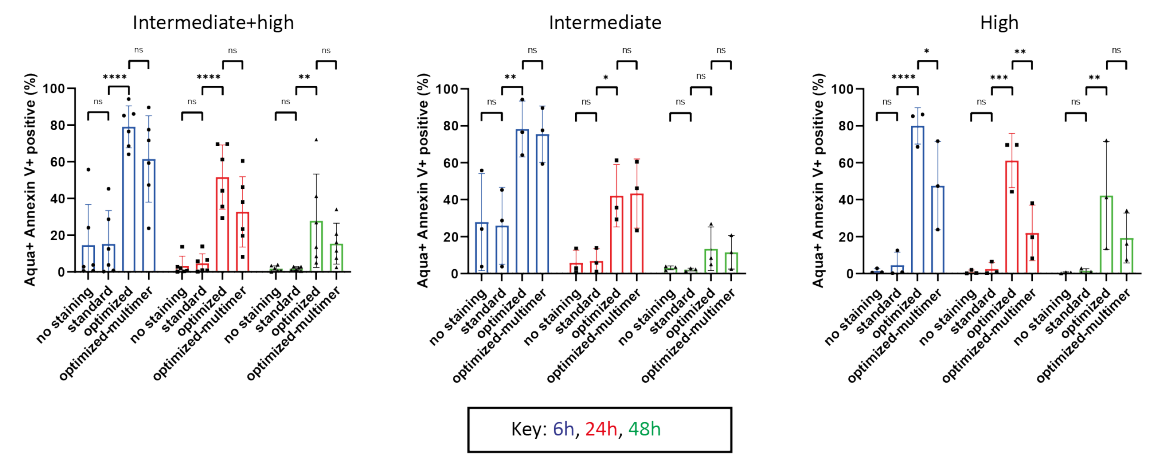

C

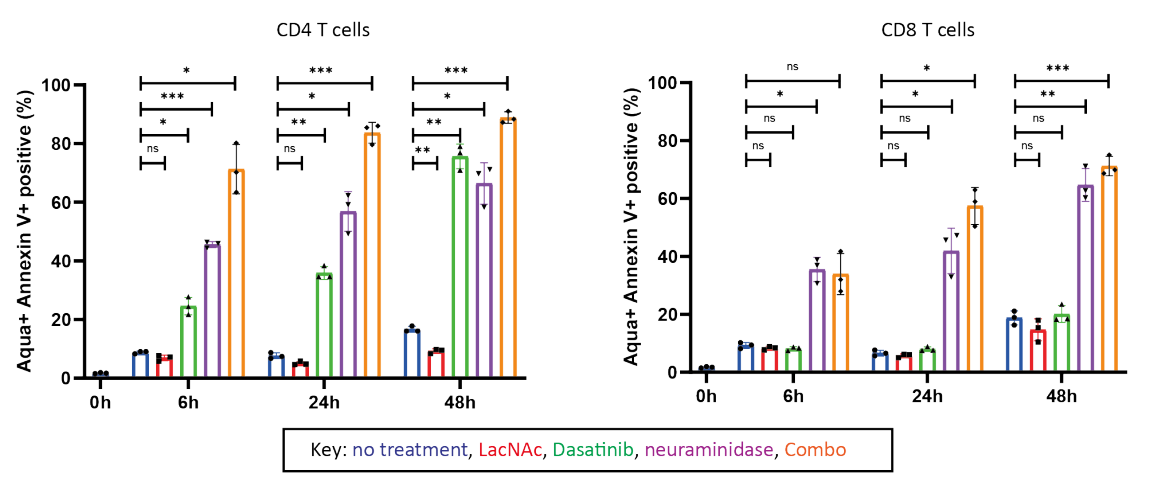

Figure 5 The optimization procedure impacts on cell viability. (A) Dot plots representing HLA-DP4/MAGE-A3 ${ }_{243-258}$ CD4 T cell clones of intermediate (left) and high staining intensity (right). Dot plots are gated on live CD3 ${ }^{+} \mathrm{CD} 4^{+}$cells. (B) Cell viability at time points 6 hours (blue), 24 hours (red) and 48 hours (green) of different HLA-DP4/MAGE-A3 ${ }_{243-258}$ CD4 T cell clones. On the left the histogram includes clones of both intermediate and high staining intensity $(n=6)$. In the center are represented only the intermediate stained clones $(n=3)$ and on the right the high stained clones $(n=3)$. Cells were gated on live, CD3 ${ }^{+} C D 4^{+}$cells. $(C)$ The effect on viability of individual or combined molecules at different time points 0 hour, 6 hours, 24 hours and 48 hours on CD4 and CD8 T cells in healthy donor PBMC.

with high MFI exhibited a reduction in the frequency of dead cells following the OSP in the absence of the multimer, suggesting that the persistence of the multimers may have a negative impact on cell viability. To evaluate which molecule in the OSP was causing an increase in cell mortality, PBMC from healthy donors were treated with LacNAc, dasatinib, neuraminidase or a combination of all three followed by culture during 6 hours, 24 hours and 48 hours before cell viability assessment. The addition of LacNAc did not affect cell survival (figure 5C) and dasatinib only impacted cell viability of CD4 $\mathrm{T}$ cells, at later time points. In contrast, neuraminidase had a strong negative impact on viability by the 6 hour time point either alone or in combination with the other compounds (figure 5C). Altogether, these results suggest that neuraminidase should be avoided to preserve the viability of specific CD4 T cells when using the OSP in settings of in vitro $\mathrm{T}$ cell expansion. The mechanism behind this loss in cell viability with the OSP seems to be linked to the improved binding of multimers to TCRs, potentially leading to activation-induced cell death (AICD).

To address this possibility, reversible multimers (NTAmers), which consist of pMHCII monomers linked by a $\mathrm{Ni}^{2+}$-nitrilotriacetic acid (NTA)-His tag-containing scaffold (figure 6A), were generated and compared with the design multimers used thus far in this work. On addition of a nontoxic low concentration of imidazole, the NTAmers decay within seconds into pMHCII monomers and PE-labeled streptavidin $\mathrm{NTA}_{4}$ molecules, causing dissociation of the pMHCII-TCR complex (figure 6B,C). HLA-DP4/MAGE-A $3_{24-258}$ cloned T cells of high staining intensity were labeled using the same conditions as in figure 5B but replacing the multimer with the NTAmer. An increase in cell viability was apparent when using 


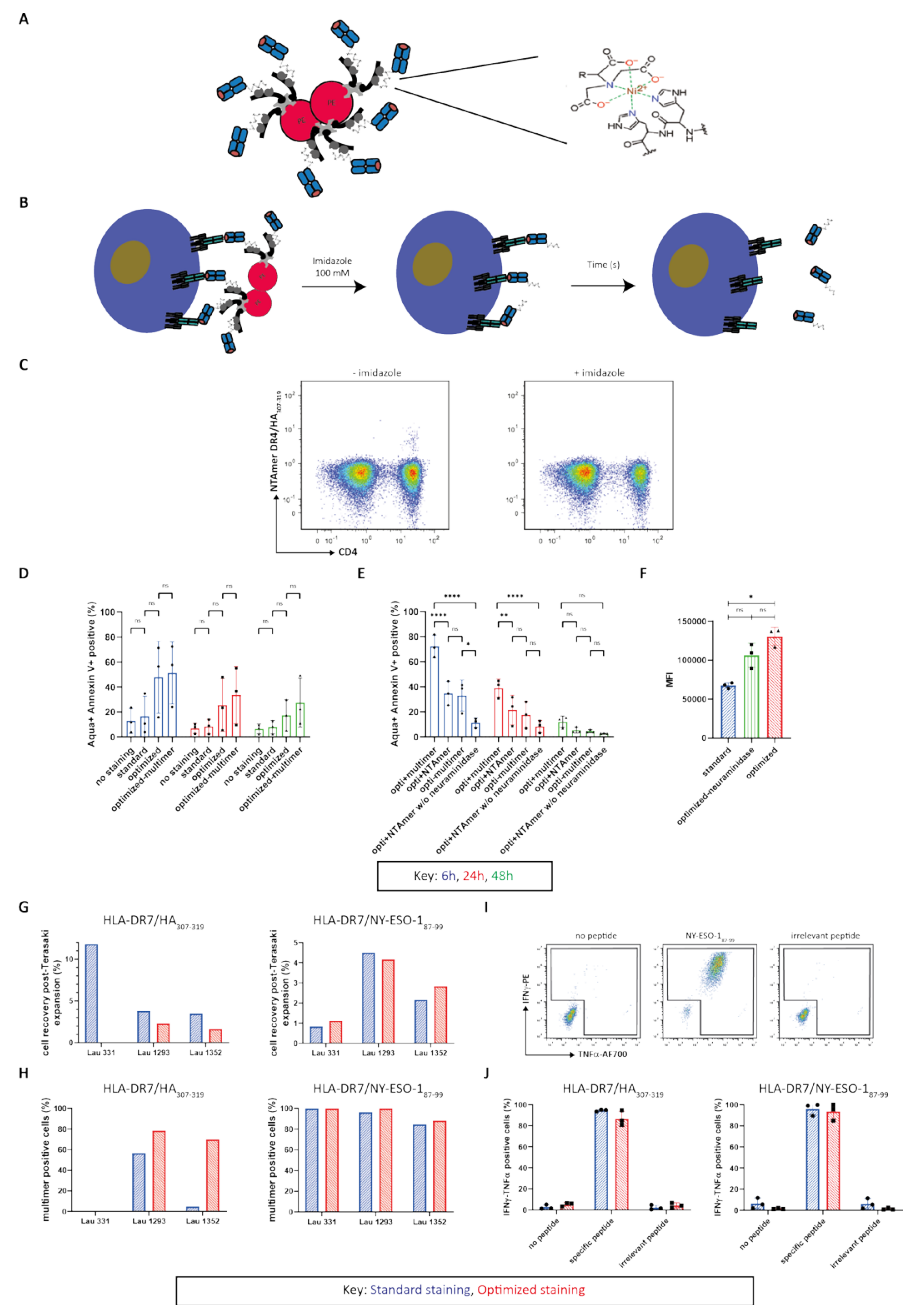

Figure 6 The use of reversible multimers to prevent activation dependent cell death. (A) Basic illustration of pMHCII NTAmers used. NTAmers are based on a fluorochrome backbone here PE (red), an average of 2-3 streptavidin complexes (grey) bound to biotin (black) linked to an imidazole-sensitive $\mathrm{Ni}^{2+}-\mathrm{NTA}_{4}$ moiety. Peptide loaded $\mathrm{MHCll}$ monomers (blue) are bound stably to the NTA tag via His tags. (B) Principle of the NTAmer staining. On addition of imidazole $(100 \mathrm{mM})$, NTAmers rapidly decay in pMHC monomers and streptavidin-PE-NTA 4 scaffolds. Monomers subsequently dissociate from cell-associated TCRs. (C) Representation of cells stained with a NTAmer (left) and the loss of the signal $1 \mathrm{~min}$ after dissociation with imidazole (right). (D) Cell viability at time points 6 hours (blue), 24 hours (red) and 48 hours (green) of HLA-DP4/MAGE-A3 ${ }_{243-258}$ clones $(n=3)$ of high staining intensity, stained using the different condition procedures indicated in the Figure. (E) Cell viability at time points 6 hours (blue), 24 hours (red) and 48 hours (green) of HLA-DP4/MAGE-A3 ${ }_{243-258}$ clones ( $n=3$ ) of high staining intensity, stained using the different condition procedures indicated in the figure. (F) Impact on multimer HLA-DR7/NY-ESO-1 ${ }_{87-99}$ staining of NY-ESO- 87- $_{87}$ ${ }_{99}$-specific clone MFI on removal of neuraminidase to improve survival. Standard protocol (blue), optimized protocol without neuraminidase (green) and full optimized protocol $(r e d)(n=3)$. (G) Cloning efficiency of CD4 T cells isolated from three patients, for both $\mathrm{HA}_{307-319}$ and NY-ESO-1 ${ }_{87-99}$ specificities, comparing cells sorted using the standard protocol (blue) and the optimized protocol (red). H) Screening of growing clones with HLA-DR7/HA ${ }_{307-319}$ or HLA-DR7/NY-ESO- ${ }_{87-99}$ multimers. Cells were initially sorted using the standard protocol (blue) or the optimized protocol (red). (I) Dot plot representation of the functionality of a representative HLA-DR7/NY-ESO- ${ }_{87-99}$ multimer positive clone sorted using the optimized staining procedure. IFN $\gamma$ and TNF $\alpha$ expression was evaluated in unstimulated cells, after stimulation with an irrelevant $\left(\mathrm{HA}_{307-319}\right.$ peptide) or the specific peptide (NY-ESO-1 87-99 peptide) (from left to right). (J) Histogram representation of the functionality of the $\mathrm{HA}_{307-319}$ or the NY-ESO-1 ${ }_{87-99}$ specific clones initially seeded by sorting using the standard protocol (blue) or the optimized protocol (red) $(n=3)$. MFI, mean fluorescence intensity; pMHCII, peptide MHC class II; TCR, T cell receptor.

NTAmers, which was comparable to that observed using the OSP in the absence of multimers (figure 6D). As expected, viability was further improved by removing neuraminidase from the staining procedure (figure 6E) with maintained superior staining intensity compared with the standard protocol (figure 6E).
To confirm the feasibility of using the above described conditions for subsequent cell culture and functional evaluation, aliquots of cells generated by the in vitro stimulation depicted in figure $3 \mathrm{G}$ were sorted with the standard protocol or the OSP and seeded in single cell cultures. Clonal efficiency was calculated for each 
patient and for both antigens (figure 6G). In regard to the NY-ESO- ${ }_{87-99}$ specificity, no major difference was observed between cells sorted with the standard protocol or the OSP. However, since the initial number of NY-ESO$1_{87-99}$-specific cells detected using the OSP in patients Lau 1293 and 1352 was on average 3.7-fold greater than with the standard protocol, many more clones could be generated (figure 6G). $\mathrm{HA}_{307-319}$-specific cells gave more positive wells with the standard protocol compared with the OSP but when screening for multimer positive clones they resulted to be non-specific for $\mathrm{HA}_{307-319}$ (figure $6 \mathrm{H}$ ), in contrast to the clones issued from the OSP sorted cells. For the NY-ESO- ${ }_{87-99}$ specificity, most clones were positively stained by the specific multimer. Finally, we excluded any functional impairment in OSP sorted clones as on specific peptide stimulation we observed significant expression of both IFN $\gamma$ and TNF $\alpha$ (figure 6I,J).

Together, these results suggest that specific $\mathrm{T}$ cell viability and functionality can be efficiently preserved by replacing standard multimers with reversible NTAmers and with the removal of neuraminidase to the optimization combo for efficient tumor-specific CD4 $\mathrm{T}$ cell expansion.

\section{DISCUSSION}

Peptide-MHC multimers have been widely used since their first description back in $1996^{22}$ and have been an invaluable tool for the detection of specific T cells. In this study, we have developed and validated novel combinatorial pMHC class II multimer staining procedures to detect human antigen-specific CD4 $\mathrm{T}$ cells, in ex-vivo patient samples. In addition, we show innovative solutions to enhance the yields of viable antigen-specific CD4 T cells after multimer-guided, flow cytometry-based cell sorting.

Despite the clear above described benefits of the OSP staining as compared with the standard procedure, a reduced cell viability was observed using OSP, which could hamper the adoption of this methodology for cell expansion in the context of ACT. We identified in the neuraminidase treatment the main cause of this cell mortality, in line with previous studies documenting its toxicity. ${ }^{23} 24$ To a lesser extent, we also observed a reduction in cell viability by dasatinib treatment. Despite this fact, dasatinib has been implemented in several tetramer staining procedures for $\mathrm{CD} 8 \mathrm{~T}$ cell identification, without mention of any negative impact on cell fitness. ${ }^{21} 2526$ Additionally, by performing the optimized staining in the absence of the multimer we observed an increase in cell viability, confirming a direct impact of the TCR-pMHC class II multimer binding on cell survival. This has already been described in antigen-specific CD8 T cells, possibly due to the AICD induced by the TCR and pMHCI interaction. ${ }^{27}$ In line with this observation in CD8 T cells, our results using the Amnis Imager show increased TCR clustering and enhanced interaction between TCR and pMHCII complexes during the OSP, supporting the concept of AICD induction also in CD4 T cells. Further, in this study, we mention for the first time the use of pMHCII NTAmers to successfully reduce cell mortality in isolated CD4 T cells. In the future, this technology could be used also for other applications, such as the measurement of TCR affinities and of the constant of TCR dissociation $\left(\mathrm{K}_{\text {off }}\right)$ as reported for CD8 T cells. ${ }^{15}$ For this endeavor, the use of the OSP might be key to ensure strong initial pMHCII NTAmer binding to TCRs of low affine tumorspecific T cells.

In an era of personalized immunotherapy, it has become vital to monitor patient responses to therapies. ${ }^{28}{ }^{29}$ The use of CD4 T cells as part of cancer therapy is coming of age, yet their monitoring remains elusive. Being able to follow the evolution of such cells is key for vaccination and adoptive cell-transfer based therapies. The large difference in percentages and phenotpye of cells detected using the standard or the optimized staining reflects discrepancies previously reported by others using other technologies. ${ }^{30}$ The combination of different peptide-MHC multimers and the evaluation of phenotypic markers is expected to generate clues as to $\mathrm{CD} 4 \mathrm{~T}$ cell differentiation and polarization, at the antigen specific level. Furthermore, TCR affinity studies will be possible, either using the 2D binding methodology or new highly sensitive methods such as the whole cell surface plasmon resonance. ${ }^{31}$ Another key aspect will be the improvement and implementation of the expanded combination of fluorochromes or metals for combinatorial multimer labeling, or the use of DNA barcodes, ${ }^{32}$ that would allow the concomitant visualization of tens of specificities as in our case and of hundreds or even thousands in one single patient sample. Moreover, the strategy to label samples with antibodies specific for both CD4 and CD8 $\mathrm{T}$ cells will allow concomitant visualization and isolation of antigen-specific cells in MHC class I and class II restricted T lymphocytes.

\section{CONCLUSION}

In conclusion, we believe this study provides significant advancement in direct human tumor-specific CD $4 \mathrm{~T}$ cell analysis by pMHC class II multimers. We propose two optimization techniques depending on the reasoning behind the detection of the specific CD4 T cells. If immunomonitoring is the focus (screening the specific cells and sequencing their TCR), then the optimization technique would include the use of LacNAc, dasatinib, neuraminidase, standard multimers as well as the secondary cross-linking Ab. However, if the focus is preserving cell viability after multimer-guided, flow cytometry-based cell sorting for further cell culture or use in cell-based therapies, then reversible NTAmers should be used and neuraminidase omitted. It remains to be shown which TCR affinity levels and TCR repertoire are detected with the optimized protocol compared with the standard one, and if the highest MFI levels correspond to T cells with the highest TCR affinity. We expect that our improved ability to dissect CD4 $\mathrm{T}$ cells at an antigen-specific level 
will decisively help with the successful targeting of these cells in immunotherapy.

Present affiliations The present affiliation of Camilla Jandus is: Department of Pathology and Immunology, Faculty of Medicine, University of Geneva, Geneva, Switzerland.

\section{Twitter Pedro J Romero @JITCancer}

Acknowledgements We would like to thank all the patients for their generous contribution and Anthony Cornu and Brigid MacKenzie for their excellent assistance. We thank Hélène Maby-El-Hajjami for the excellent assistance with clinical data of the patients.

Funding This study was supported in part by grants from the MEDIC Foundation, the Helmut Horten Foundation, the Swiss National Science Foundation (PR00P3_179727), the Ludwig Institute for Cancer Research and the Stiftung für Krebsbekämpfung. AC is recipient of an ISREC fellowship. PJR was supported in part by grants from the SNSF 31003A_156469 and 310030_182735.

Competing interests None declared.

Patient consent for publication Not required.

Ethics approval Patients' samples were obtained from the Department of Oncology, University Hospital (CHUV), Lausanne, Switzerland, on written informed consent based on the study protocol (NCT00112242) approved by the IRB and Swissmedic.

Provenance and peer review Not commissioned; externally peer reviewed.

Data availability statement Data are available on reasonable request. All data relevant to the study are included in the article or uploaded as supplementary information.

Open access This is an open access article distributed in accordance with the Creative Commons Attribution Non Commercial (CC BY-NC 4.0) license, which permits others to distribute, remix, adapt, build upon this work non-commercially, and license their derivative works on different terms, provided the original work is properly cited, appropriate credit is given, any changes made indicated, and the use is non-commercial. See http://creativecommons.org/licenses/by-nc/4.0/.

\section{ORCID iDs}

Georg Alexander Rockinger http://orcid.org/0000-0002-0037-7356

Camilla Jandus http://orcid.org/0000-0002-7405-5747

\section{REFERENCES}

1 Restifo NP, Dudley ME, Rosenberg SA. Adoptive immunotherapy for cancer: harnessing the T cell response. Nat Rev Immunol 2012;12:269-81.

2 Spitzer MH, Carmi Y, Reticker-Flynn NE, et al. Systemic immunity is required for effective cancer immunotherapy. Cell 2017;168:487-502.

3 Baumgaertner P, Costa Nunes C, Cachot A, et al. Vaccination of stage III/IV melanoma patients with long NY-ESO-1 peptide and $\mathrm{CpG}-\mathrm{B}$ elicits robust $\mathrm{CD}^{+}$and $\mathrm{CD}^{+}{ }^{+} \mathrm{T}-$ cell responses with multiple specificities including a novel DR7-restricted epitope. Oncoimmunology 2016;5:e1216290.

4 Ott PA, Hu Z, Keskin DB, et al. An immunogenic personal neoantigen vaccine for patients with melanoma. Nature 2017;547:217-21.

5 Bentzen AK, Marquard AM, Lyngaa R, et al. Large-Scale detection of antigen-specific T cells using peptide-MHC-I multimers labeled with DNA barcodes. Nat Biotechnol 2016;34:1037-45.

6 Cole DK, Pumphrey NJ, Boulter JM, et al. Human TCR-binding affinity is governed by MHC class restriction. $J$ Immunol 2007;178:5727-34.

7 Hamad AR, O'Herrin SM, Lebowitz MS, et al. Potent T cell activation with dimeric peptide-major histocompatibility complex class II ligand: the role of CD4 coreceptor. J Exp Med 1998;188:1633-40.

8 Crawford F, Kozono $\mathrm{H}$, White J, et al. Detection of antigen-specific T cells with multivalent soluble class II MHC covalent peptide complexes. Immunity 1998;8:675-82.
9 Jönsson P, Southcombe JH, Santos AM, et al. Remarkably low affinity of CD4/peptide-major histocompatibility complex class II protein interactions. Proc Natl Acad Sci U S A 2016;113:5682-7.

10 Schmidt J, Dojcinovic D, Guillaume P, et al. Analysis, Isolation, and Activation of Antigen-Specific CD4(+) and CD8(+) T Cells by Soluble MHC-Peptide Complexes. Front Immunol 2013;4:218.

11 Kalandadze A, Galleno M, Foncerrada L, et al. Expression of recombinant HLA-DR2 molecules. replacement of the hydrophobic transmembrane region by a leucine zipper dimerization motif allows the assembly and secretion of soluble DR alpha beta heterodimers. Biol Chem 1996;271:20156-62.

12 Ayyoub M, Dojcinovic D, Pignon P, et al. Monitoring of NY-ESO-1 specific CD4+ T cells using molecularly defined MHC class II/Histag-peptide tetramers. Proc Natl Acad Sci U S A 2010;107:7437-42.

13 Fremont $\mathrm{DH}$, Hendrickson WA, Marrack P, et al. Structures of an $\mathrm{MHC}$ class II molecule with covalently bound single peptides. Science 1996;272:1001-4.

14 Uchtenhagen H, Rims C, Blahnik G, et al. Efficient ex vivo analysis of CD4+ T-cell responses using combinatorial HLA class II tetramer staining. Nat Commun 2016;30:12614.

15 Hebeisen M, Schmidt J, Guillaume P, et al. Identification of rare highavidity, Tumor-Reactive CD8+ T cells by monomeric TCR-Ligand OffRates measurements on living cells. Cancer Res 2015;75:1983-91.

16 Rabinovich GA, Toscano MA. Turning 'sweet' on immunity: galectinglycan interactions in immune tolerance and inflammation. Nat Rev Immunol 2009;9:338-52.

17 Demotte N, Stroobant V, Courtoy PJ, et al. Restoring the association of the T cell receptor with CD8 reverses anergy in human tumorinfiltrating lymphocytes. Immunity 2008;28:414-24.

18 Petit A-E, Demotte N, Scheid B, et al. A major secretory defect of tumour-infiltrating $T$ lymphocytes due to galectin impairing LFA-1mediated synapse completion. Nat Commun 2016;7:12242.

19 Lu Y-C, Parker LL, Lu T, et al. Treatment of patients with metastatic cancer using a major histocompatibility complex class II-restricted Tcell receptor targeting the cancer germline antigen MAGE-A3. J Clin Oncol 2017;35:3322-9.

20 Mekala DJ, Geiger TL. Functional segregation of the TCR and antigen-MHC complexes on the surface of CTL. J Immunol 2003;171:4089-95.

21 Uchtenhagen H, Rims C, Blahnik G, et al. Efficient ex vivo analysis of CD4+ T-cell responses using combinatorial HLA class II tetramer staining. Nat Commun 2016;7:12614.

22 Altman JD, Reay PA, Davis MM. Formation of functional peptide complexes of class II major histocompatibility complex proteins from subunits produced in Escherichia coli. Proc Natl Acad Sci U S A 1993;90:10330-4.

23 Kelly R, Greiff D. Toxicity of pneumococcal neuraminidase. Infect Immun 1970;2:115-7.

29 Weiss L, Editorial G. Neuraminidase, sialic acids, and cell interactions. J Natl Cancer Inst 1973;50:3-19.

25 Dolton G, Zervoudi E, Rius C, et al. Optimized peptide-MHC multimer protocols for detection and isolation of autoimmune T-cells. Front Immunol 2018;9:1378.

26 Jansen DTSL, Ramnoruth N, Loh KL, et al. Flow cytometric clinical Immunomonitoring using Peptide-MHC class II tetramers: optimization of methods and protocol development. Front Immunol . 2018;9.

27 Xu XN, Purbhoo MA, Chen N, et al. A novel approach to antigenspecific deletion of CTL with minimal cellular activation using alpha3 domain mutants of MHC class I/peptide complex. Immunity 2001;14:591-602.

28 Hollingsworth RE, Jansen K. Turning the corner on therapeutic cancer vaccines. NPJ Vaccines 2019;4:7.

29 Hilf N, Kuttruff-Coqui S, Frenzel K, et al. Actively personalized vaccination trial for newly diagnosed glioblastoma. Nature 2019;565:240-5.

30 Martinez RJ, Andargachew R, Martinez HA, et al. Low-Affinity CD4+ $\mathrm{T}$ cells are major responders in the primary immune response. Nat Commun 2016;7:13848.

31 Soler M, Li X, John-Herpin A, et al. Two-Dimensional label-free affinity analysis of tumor-specific CD8 T cells with a biomimetic plasmonic sensor. ACS Sens 2018;3:2286-95.

32 Bentzen AK, Hadrup SR. Evolution of MHC-based technologies used for detection of antigen-responsive T cells. Cancer Immunol Immunother 2017;66:657-66. 